\title{
Evanescent wave imaging of adsorbed protein layers
}

An evanescent wave arises at the interface of two media when light propagates from a more to a less-dense medium under total internal reflection. The wave is distributed over a superficial area because its amplitude decays exponentially with distance from the interface. The evanescent wave intensity at the interface can be larger than that of the incident beam. Evanescent waves have widespread current use in the imaging of chemical, bio-chemical and biological phenomenon. For example, an evanescent wave is responsible for fluorophore excitation in total internal reflection fluorescence microscopy, which is used to visualize single-molecules on the surface of living cells, and can detect cell membranes in micro-domains. An interesting scientific question is the possibility of directly using evanescent waves to image a monolayer, and how to improve the image contrast. The authors have observed an adsorbed protein layer using evanescent wave imaging and used both experimental and theoretical techniques in their investigation.

The authors first considered the possibility of visualizing a protein monolayer at a glass-liquid interface. A plane light wave and classic theoretical simulation were used to investigate the evanescent wave behavior. The resulting image of the protein monolayer demonstrated the difficulty in such visualization, due to low image contrast (about $10^{-5}$ ) between the protein layer and glass substrate.

Evanescent waves arise from the oblique incidence angle, which would change the polarization state of the reflected light wave. Thus, a polarized beam was applied. The optimized polarization setting resulted in a 100 times improvement in image contrast. However, this was still insufficient to adequately visualize the protein monolayer.

A surface-enhanced medium (e.g. a gold layer) was used at the glass interface, to provide a surface-enhanced effect for evanescent wave imaging. The gold layer thickness was selected and using an optimized incident angle, the image contrast increased by about $10^{7}$ of that without the gold layer. The adsorbed protein monolayer on the gold surface was satisfactorily visualized.

Finally, the polarization state was optimized and the maximum image contrast increased slightly. However, this improvement was more apparent at image regions which neighbored those of maximum contrast.

In this study, the effect on image contrast of incident angle, gold layer and polarization were also theoretically simulated. The authors observed that the angle of incidence could be optimized to achieve a maximum imaging contrast and a 100 times increase could be obtained when an optimized polarization was applied as well. When the gold layer surface enhancement effect was incorporated, a dramatic contrast increase of 107 was observed. Interestingly, the image contrasts of the gold-induced imaging with and without polarization optimization were almost identical. This was vastly different when imaging was performed without the gold layer. The gold layer appeared to produce a surface enhanced effect on image contrast, while at the same time having a depolarization effect. Thus, polarization optimization seems no longer to be necessary in imaging applications using a gold layer.

Image contrast is very sensitive to the thickness of the gold layer and also very sensitive to the gold layer refractive index, which was not simulated in this study. In practice, the gold layer thickness and refractive index is much harder to control than in simulation, so experimental measurements were generally carried out in non-optimized conditions. The real setting in experiments becomes the neighboring region of optimization, where polarization played an important role in improving image contrast (such as Figure 1).

"This paper emphasizes the visualization of a protein monolayer by evanescent wave imaging and its optimization of conditions. The theoretical simulation and experiment results are of academic and practical significance," said one journal reviewer. A series of papers about biosensor applications with evanescent wave imaging have been published by the authors in various scientific journals, which offer us new prospects for developing evanescent wave imaging and associated applications.
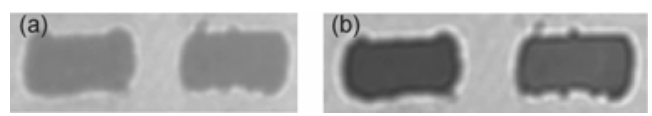

Figure 1 Image of immunoglobulin adsorbed layer. (a) Image of an adsorbed layer of immunoglobulin $\mathrm{G}(\mathrm{IgG})$ on a gold-glass substrate, by evanescent wave imaging without polarization. (b) A similar image obtained using optimized polarization.

The authors are affiliated with the Biophotonics group at the National Microgravity Laboratory (NML) of the Institute of Mechanics at the Chinese Academy of Sciences (IMech, CAS). This group is conducting research predominantly on protein microarray biosensors and applications, spatially-resolved and time-resolved ellipsometric techniques, and the characterization of biomaterials with ellipsometry and SNOM. Funding from the National Basic Research Program of China (2009CB320300) and the National High Technology Research and Development Program of China (2008AA02Z419), supported this research.

See the article: Liu L, Niu Y, Chen S, et al. Optimization of evanescent wave imaging for visualization of protein adsorption layer. Sci China Phys Mech Astron, 2010, 53, 1805-1810 